Pacific Journal of Mathematics

HOMOLOGY OF A GROUP EXTENSION 


\section{HOMOLOGY OF A GROUP EXTENSION}

\section{YASUTOSHI NomURA}

A topological method has been used by Ganea to derive the homology exact sequence of a central extension. In the same spirit a homology exact sequence is constructed for a group extension with certain homological restrictions. An immediate consequence is an exact sequence of Kervaire which is of some significance in algebraic $K$-theory.

Let

$$
1 \longrightarrow N \rightarrow G \longrightarrow Q \longrightarrow 1
$$

be an extension of groups. Each element $g$ of $G$ induces an automorphism $\theta(g): N \rightarrow N$ via $\theta(g) n=g n g^{-1}$ for $n \in N$. In what follows we denote by $H_{k}(G)$ the $k$ th homology group of $G$ with coefficients in the additive group of integers $Z$, on which $G$ operates trivially. Let $\Gamma_{k}$ denote the subgroup of $H_{k}(N)$ generated by $\theta(g)_{*} c-c, c \in H_{k_{k}}(N), g \in$ $G$. We say that $G$ operates trivially on $H_{k}(N)$ if $\Gamma_{k}=\{0\}$. Let $\bar{N} \widetilde{\times}$ $G$ be the semi-direct product of $N$ and $G$ with respect to the operation $\theta(g)$ and let $P_{k}$ denote the kernel of $\pi_{*}: H_{k}(N \widetilde{\times} G) \rightarrow H_{k}(G)$, where $\pi: N \widetilde{\times} G \rightarrow G$ is given by $\pi(n, g)=g$. We shall prove

Theorem 1. Suppose $n=1$ or $H_{k}(N)=0$ for $1 \leqq k \leqq n-1(n \geqq 2)$. Then there exists an exact sequence

$$
\begin{aligned}
P_{2 n} & \longrightarrow H_{2 n}(G) \longrightarrow H_{2 n}(Q) \longrightarrow P_{2 n-1} \longrightarrow \cdots \longrightarrow P_{n+1} \longrightarrow H_{n+1}(G) \\
& \longrightarrow H_{n+1}(Q) \longrightarrow H_{n}(N) / \Gamma_{n} \longrightarrow H_{n}(G) \longrightarrow H_{n}(Q) \longrightarrow 0 .
\end{aligned}
$$

Further assume $G$ operates trivially on $H_{n}(N)$ and that $H_{1}(Q)=0$. Then there exists an exact sequence

$$
H_{n+1}(N) \longrightarrow H_{n+1}(G) \longrightarrow H_{n+1}(Q) \longrightarrow H_{n}(N) \longrightarrow H_{n}(G) \longrightarrow H_{n}(Q) \longrightarrow 0 \text {. }
$$

We note that the first part of Theorem 1 for $n=1$ is just Theorem 3.1 of [7].

Now we call an epimorphism $f: H \rightarrow H^{\prime}$ central if $\operatorname{Ker} f$ is contained in the center of $H$. Let

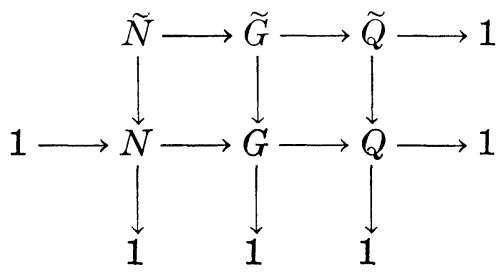


be a commutative diagram of groups and homomorphisms such that the rows and columns are exact.

Theorem 2. In the situation (2) suppose $\widetilde{N} \rightarrow N$ and $\widetilde{Q} \rightarrow Q$ are central, $H_{1}(\widetilde{N})=H_{1}(\widetilde{Q})=H_{1}(N)=0$ and that $G$ operates trivially on $H_{2}(N)$. Then there exists an exact sequence

$$
H_{3}(\tilde{N}) \longrightarrow H_{3}(\widetilde{G}) \longrightarrow H_{3}(\widetilde{Q}) \longrightarrow H_{2}(N) \longrightarrow H_{2}(G) \longrightarrow H_{2}(Q) \longrightarrow 0
$$

As a special case of Theorem 2 we obtain Prop. 2 of Kervaire [6] (cf. [1]).

THEOREM 3. In (1) let $Q$ be the additive group of integers $Z$ and let $e$ be an element of $G$ which maps to $+1 \in Z$. Then there exists a long exact sequence

$$
\begin{aligned}
\cdots \longrightarrow H_{k}(N) \stackrel{1-O(e)_{*}}{\longrightarrow} H_{k}(N) \longrightarrow H_{k}(G) \longrightarrow H_{k-1}(N) & \\
\cdots \longrightarrow H_{1}(N) & \longrightarrow H_{1}(G) \longrightarrow Z \longrightarrow 0 .
\end{aligned}
$$

1. Topological preliminaries. In this section two lemmas are established which play a vital role in the proofs of Theorems. We work in the category of based spaces which have the homotopy type of a $C W$ complex. We use the notation $\vee$ for path-composition. The multiplication of elements of fundamental groups are indicated by juxtaposition. Given a map $f: X \rightarrow Y$ we denote by $f_{\sharp}$ the homomorphism induced on fundamental groups.

Given a map $p: E \rightarrow B$, let $\rho: E_{p} \rightarrow E$ denote the fibre of $p$, that is, $E_{p}=\left\{(x, \beta) \in E \times B^{I} ; \beta(0)=*, \beta(1)=p(x)\right\}$ with $\rho(x, \beta)=x$, where * stands for the base point. $\Omega B$, the space of loops on $B$, acts on $E_{p}$ through $\mu: \Omega B \times E_{p} \rightarrow E_{p}, \mu(\omega,(x, \beta))=(x, \omega \vee \beta)$. We define

$$
\tilde{\mu}: \tilde{H}_{0}(\Omega B) \otimes H_{k}\left(E_{p}\right) \longrightarrow H_{k}\left(E_{p}\right)
$$

to be the composite

$$
\widetilde{H}_{0}(\Omega B) \otimes H_{k}\left(E_{p}\right) \subset H_{0}(\Omega B) \otimes H_{k}\left(E_{p}\right) \longrightarrow H_{k}\left(\Omega B \times E_{p}\right) \stackrel{\mu_{*}}{\longrightarrow} H_{k}\left(E_{p}\right),
$$

where the middle arrow comes from Künneth theorem and $\widetilde{H}_{0}(\Omega B)$ may be identified with the subring of the integral group ring of $\pi_{1}(B)$ generated by $\omega-1, \omega \in \pi_{1}(B)$.

Now let $p$ be a Hurewicz fibration with fibre inclusion $i: F \rightarrow E$. As shown by Eckmann-Hilton [2; Prop. 3.10 and Theorem 3.11], the above $\mu$ determines an action of $\Omega B$ on $F$, which is denoted by the same letter $\mu$. We say that $\pi_{1}(B)$ operates trivially on $H_{k}(F)$ if the above $\tilde{\mu}$ is trivial.

Let $S$ denote the suspension functor and let $C_{p}$ denote the cofibre 
of $p$, that is, $C_{p}=B \bigcup_{p} C E$ (with $(x, 1)$ and $p(x)$ identified). Let

$$
\sigma: S F \longrightarrow C_{p}
$$

denote the canonical embedding defined by $\sigma(x, t)=(x, t) \in C E, x \in F$, $0 \leqq t \leqq 1$.

Lemma 1.1. Suppose that $B$ is path-connected and that $F$ is homology $(n-1)$-connected, $n \geqq 1$. Then $\sigma$ is homology $(n+1)$-connected and the sequence

$$
\widetilde{H}_{0}(\Omega B) \otimes H_{n}(F) \stackrel{\tilde{\mu}}{\longrightarrow} H_{n}(F) \stackrel{\sigma_{*}}{\longrightarrow} H_{n+1}\left(C_{p}\right) \longrightarrow 0
$$

is exact.

Proof. According to Ganea [3; Theorem 1.1], the extension $r: C_{i} \rightarrow$ $B$ of $p$ to $E \cup C F$ has the fibre equivalent to $\Omega B * F$, which is $n$-connected. Thus the argument in [3; Theorem 2.2] is valid in our case, hence there is an exact sequence

$$
H_{k}(\Omega B * F) \stackrel{H_{*}}{\longrightarrow} H_{k}(S F) \stackrel{\sigma_{*}}{\longrightarrow} H_{k}\left(C_{p}\right) \longrightarrow H_{k-1}(\Omega B * F)
$$

for $k \leqq n+1$, where $H: \Omega B * F \rightarrow S F$ is the map obtained from $\mu$ by the Hopf construction. It is immediate that $H_{*}$ coincides with $\tilde{\mu}$ on $H_{n+1}(\Omega B * F) \cong \widetilde{H}_{0}(\Omega B) \otimes H_{n}(F)$, which proves the assertion.

COROLlaRY 1.2. In addition to the assumption of Lemma 1.1, suppose further $\pi_{1}(B)$ operates trivially on $H_{n}(F)$ and that $H_{1}(B)=0$. Then $\sigma$ is homology $(n+2)$-connected.

Proof. Since $C_{i}$ is the double mapping cylinder of $* \leftarrow F \stackrel{i}{\longrightarrow} E$, $r: C_{i} \leftarrow B$ is homotopically equivalent to the Whitney join

$$
p_{B} \oplus p: P B \oplus E \longrightarrow B
$$

of the path-fibration $p_{B}: P B \rightarrow B$ and $p$ ([5]. For the notation see [7]). It follows from the construction of a lifting function of Whitney join (See Hall [5; §3]) that, in $p_{B} \oplus p, \Omega B$ operates on $\Omega B * F$ through $\nu: \Omega B \times(\Omega B * F) \rightarrow \Omega B * F$ as the join of the actions in each fibration; thus, $\nu(\alpha,(1-t) B \oplus t x)=(1-t)\left(\beta \vee \alpha^{-1}\right) \oplus t \mu(\alpha, x)$ for $\alpha, \beta \in \Omega B, x \in$ $F, 0 \leqq t \leqq 1$. Consequently, $\widetilde{\nu}$ is given by

$$
\tilde{\nu}((\alpha-1) \otimes((\beta-1) \otimes c))=(\beta-1)\left(\alpha^{-1}-1\right) \otimes c
$$

under the assumption $\tilde{\mu}((\alpha-1) \otimes c)=0$.

Applying Lemma 1.1 to $p_{B} \oplus p$, we get an exact sequence $\widetilde{H}_{0}(\Omega B) \otimes H_{n+1}(\Omega B * F) \stackrel{\check{\Sigma}}{\longrightarrow} H_{n+1}(\Omega B * F) \longrightarrow H_{n+2}\left(C_{p_{B} \oplus p}\right) \longrightarrow 0$. 
Since $\pi_{1}(B)=\left[\pi_{1}(B), \pi_{1}(B)\right]$ by assumption and since the identity

$$
\begin{aligned}
\alpha \beta \alpha^{-1} \beta^{-1}-1= & \left(\alpha \beta \alpha^{-1}-1\right)\left(\beta^{-1}-1\right)+(\alpha-1)\left(\beta \alpha^{-1}-1\right) \\
& -\left(\beta \alpha^{-1}-1\right)(\alpha-1)-(\beta-1)\left(\beta^{-1}-1\right)
\end{aligned}
$$

holds in the integral group ring of $\pi_{1}(B)$ we may infer that $\widetilde{\nu}$ is epic. This implies that $H_{n+2}\left(C_{p_{B} \oplus p}\right) \cong H_{n+2}\left(C_{r}\right)=0$. Since $C_{\sigma}$ is of the same homotopy type as $C_{r}$ by [3; Prop. 1.6], we see that $\sigma$ is homology $(n+2)$-connected.

Next consider an extension of groups (1). We may construct a Hurewicz fibration $p: E \rightarrow B$ of aspherical spaces with fibre inclusion $i: F \rightarrow E$ so that the sequence

$$
1 \longrightarrow \pi_{1}(F) \stackrel{i_{\sharp}}{\longrightarrow} \pi_{1}(E) \stackrel{p_{\sharp}}{\longrightarrow} \pi_{1}(B) \longrightarrow 1
$$

coincides with the given extension (1). We shall relate $\theta(g)_{*}$ to the action $\tilde{\mu}$ of $\pi_{1}(B)$ on $H_{*}\left(F^{\prime}\right)$.

As in the beginning of this section, we may replace $i: F \rightarrow E$ by $\rho: E_{p} \rightarrow E$. Let $g \in G=\pi_{1}(E)$ and let $\overline{\theta(g)}$ denote a map $\left(E_{p}, *\right) \rightarrow$ $\left(E_{p}, *\right)$ induced by $\theta(g)$. Take $\alpha:(I, \dot{I}) \rightarrow(E, *)$ which represents $g$. Define a path $\Delta(\alpha)$ in $E_{p}$ joining $(*, *)$ with $(*, p \alpha \vee *)$ by setting

$$
\begin{aligned}
\Delta(\alpha)(t) & =\left(\alpha(t), \bar{\alpha}_{t}\right), \\
\bar{\alpha}_{t}(s) & =\left\{\begin{array}{l}
p \alpha(2 s) \\
p \alpha(t)
\end{array}\right.
\end{aligned}
$$$$
\begin{aligned}
& 0 \leqq 2 s \leqq t \\
& t \leqq 2 s \leqq 2
\end{aligned}
$$

$\mu$ defines a map $\mu(\alpha):\left(E_{p}, *\right) \rightarrow\left(E_{p},(*, p \alpha \vee *)\right)$ given by

$$
\mu(\alpha)(x, \beta)=\mu(p \alpha ;(x, \beta))=(x, p \alpha \vee \beta) .
$$

Since $E_{p}$ has a non-degenerate base point [8], we obtain a map

$$
\overline{\mu(\alpha)}:\left(E_{p}, *\right) \longrightarrow\left(E_{p}, *\right)
$$

which is $\Delta(\alpha)$-homotopic to $\mu(\alpha)$.

LEMMA 1.5. There is a based homotopy between $\overline{\mu(\alpha)}$ and $\overline{\theta(g)}$.

Proof. It suffices to prove that, for each loop $\omega:(I, \dot{I}) \rightarrow\left(E_{p}, *\right)$, we have $\overline{\mu(\alpha)_{\sharp}} \omega=\overline{\theta(g)_{\xi}} \omega$. We see that $\Delta(\alpha) \vee \mu(\alpha) \omega \vee \Delta(\alpha)^{-1}:(I, \dot{I}) \rightarrow$ $\left(E_{p}, *\right)$ is $\Delta(\alpha)$-homotopic to $\mu(\alpha) \omega$ and that $\rho\left(\Delta(\alpha) \vee \mu(\alpha) \omega \vee \Delta(\alpha)^{-1}\right)=$ $\alpha \vee \rho \omega \vee \alpha^{-1}$. Thus, by [8; Lemma 7.3.2(b)],

$$
\overline{\mu(\alpha)} \omega \simeq \Delta(\alpha) \vee \mu(\alpha) \omega \vee \Delta(\alpha)^{-1}
$$

and, since $\rho \overline{\theta(g)} \omega=\alpha \vee \rho \omega \vee \alpha^{-1}$ by definition and since $\rho_{\sharp}$ is monic, it follows that $\Delta(\alpha) \vee \mu(\alpha) \omega \vee \Delta(\alpha)^{-1} \simeq \overline{\theta(g)} \omega$. These yield $\overline{\mu(\alpha)} \omega \simeq$ $\overline{\theta(g)} \omega$, as desired. 
CoROLlaRY 1.4. $\tilde{\mu}\left(\left(p_{\ddagger} \alpha-1\right) \otimes c\right)=\theta(g)_{*} c-c$ for $c \in H_{k}(F), \alpha \in \pi_{1}(E)$. For, we have

$$
\begin{aligned}
\tilde{\mu}\left(\left(p_{\sharp} \alpha-1\right) \otimes c\right) & =\mu_{*}\left(p_{\sharp} \alpha, c\right)-c=\mu(\alpha)_{*} c-c \\
& \left.=\overline{\mu(\alpha)})_{*} c-c=\overline{\theta(g}\right)_{*} c-c \\
& =\theta(g)_{*} c-c
\end{aligned}
$$

2. Proof of Theorem 1. Let $p: E \rightarrow B$ be a fibration with fibre inclusion $i: F \rightarrow E$ which is used in the proof of Lemma 1.3. Introduce the following commutative diagram

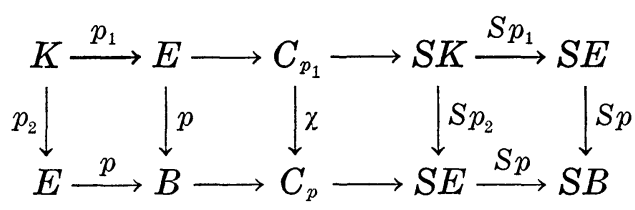

in which the square in the left corner is the pull-back of $p$ by $p, \chi$ is induced by it and the rows are Puppe sequences for $p_{1}$ and $p$. Since $F * F$ is $2 n$-connected, it follows that $\chi$ is homology $(2 n+1)$ connected (cf. [7; 1.1 and 1.2]).

Since $p_{1}$ admits a cross-section, $H_{k}\left(C_{p_{1}}\right)$, identified with a subgroup of $H_{k-1}(K)$, coincides with the kernel of $p_{1^{*}}: H_{k-1}(K) \rightarrow H_{k-1}(E)$. As shown in [7; 3.1], $\pi_{1}(K) \cong N \widetilde{\times} G$ and, under this isomorphism, $p_{1 \sharp}(n, g)=g$, which implies that $\operatorname{Ker} p_{1^{*}}=P_{k-1}$.

Observe that the composite $S F \stackrel{\sigma}{\longrightarrow} C_{p} \longrightarrow S E$ coincides with $S_{I}$. Lemma 1.1 applied to $p$ yields an exact sequence

$$
\widetilde{H}_{0}(\Omega B) \otimes H_{n}(F) \stackrel{\tilde{\mu}}{\longrightarrow} H_{n+1}(S F) \stackrel{\sigma_{*}}{\longrightarrow} C_{n+1}\left(C_{p}\right) \longrightarrow 0
$$

and bijections $\sigma_{*}: H_{k}(S F) \rightarrow H_{k}\left(C_{p}\right)$ for $k \leqq n$. It follows from Corollary 1.4 that $\operatorname{Im} \tilde{\mu}=\Gamma_{k}$, hence $H_{n+1}\left(C_{p}\right) \cong H_{n}(N) / \Gamma_{n}$. Thus we obtain an exact sequence stated in Theorem 1 , which completes the proof of the first part of Theorem 1.

Further assume $H_{1}(B)=0$ and that $\Gamma_{n}=0$; then, by Corollary $1.2, \sigma_{*}: H_{n+2}(S F) \rightarrow H_{n+2}\left(C_{p}\right)$ is epic, hence there is an exact sequence

$$
H_{n+1}(F) \longrightarrow H_{n+1}(E) \longrightarrow H_{n+1}(B) \longrightarrow H_{n}(F) \longrightarrow H_{n}(E) \longrightarrow H_{n}(B) \longrightarrow 0 \text { . }
$$

which yields the second part of Theorem 1 .

3. Proof of Theorem 2. First we shall prove

Lemma 3.1. (Kervaire [6; Lemma 3]) Let $1 \rightarrow N \rightarrow G \rightarrow Q \rightarrow 1$ be a central extension of groups. If $H_{k}(G)=0$ for $1 \leqq k \leqq n$, then the sequence 


$$
\begin{aligned}
& H_{n+2}(G) \longrightarrow H_{n+2}(Q) \longrightarrow H_{n+2}(N, 2 ; Z) \longrightarrow H_{n+1}(G) \\
& \longrightarrow H_{n+1}(Q) \longrightarrow H_{n+1}(N, 2 ; Z)
\end{aligned}
$$

is exact. In particular, if $H_{1}(G)=0$, then $H_{3}(G) \rightarrow H_{3}(Q)$ is epic and $\mathrm{H}_{2}(G) \rightarrow H_{2}(Q)$ is monic.

Proof. Let $F \stackrel{i}{\longrightarrow} E \stackrel{p}{\longrightarrow} B$ be as in the proof of Lemma 1.3. As shown by Ganea [4], $p$ is homotopically equivalent to the principal fibration $E_{\phi} \rightarrow B$ induced by a map $\phi: B \rightarrow C=K(N, 2)$. Let $\tilde{\phi}: C_{p} \rightarrow$ $C$ denote the canonical extensin of $\phi$ to $B \mathrm{U}_{p} C E$. By [3; Theorem 1.1] the fibre of $\tilde{\phi}$ is equivalent to $E * \Omega C$, which is $(n+2)$-connected. This implies that $\tilde{\phi}$ is $(n+3)$-connected. Thus, by replacing $H_{k}\left(C_{p}\right)$ for $k \leqq n+2$ by $H_{k}(C)$ in the Puppe sequence of $p$, there is obtained the desired exact sequence. The second part follows from the fact that $H_{3}(N, 2 ; Z)=0$.

We now proceed to the proof of Theorem 2. Let $\bar{N}$ denote the kernel of $\widetilde{G} \rightarrow \widetilde{Q}$ in (2). Then the diagram (2) may be enlarged to the following

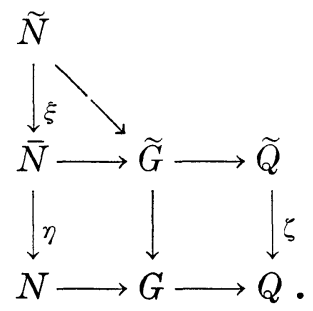

Note that $\xi$ and $\eta$ are epic, hence central with $H_{1}(\bar{N})=0$.

Introduce the commutative diagram

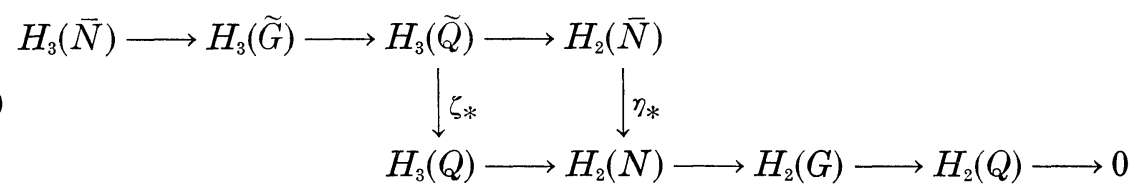

where $\zeta_{*}$ is epic and $\eta_{*}$ is monic by Lemma 3.1. Hence it follows from naturality of action that $\widetilde{G}$ operates trivially on $H_{2}(\bar{N})$. Applying Theorem 1 to the extensions $1 \rightarrow \bar{N} \rightarrow \widetilde{G} \rightarrow \widetilde{Q} \rightarrow 1$ and $1 \rightarrow N \rightarrow G \rightarrow$ $Q \rightarrow 1$, we see that the rows of (4) are exact. Since $\xi_{*}: H_{3}(\widetilde{N}) \rightarrow H_{3}(\bar{N})$ is epic by Lemma 3.1, we may conclude that the sequence stated in Theorem 2 is exact.

4. Proof of Theorem 3. We may take the circle $S^{1}$ for $B$ in the fibration $F \stackrel{i}{\longrightarrow} E \stackrel{p}{\longrightarrow} B$ which realizes (1). We use the Wang sequence for $p$ which is found in Spanier [8; 8.5.5]. There are fibre homotopy equivalences 


$$
f_{-}: C_{-} S^{0} \times F \longrightarrow p^{-1}\left(C_{-} S^{0}\right), g_{+}: p^{-1}\left(C_{+} S^{0}\right) \longrightarrow C_{+} S^{0} \times F
$$

such that $f_{-} \mid y_{0} \times F$ is homotopic to the map $\left(y_{0}, x\right) \rightarrow x$ and $g_{+} \mid F$ is homotopic to the map $x \rightarrow\left(y_{0}, x\right)$, where $y_{0}$ denotes the base point corresponding to $\{0\} \in S^{0}$ and where $C_{-} S^{0}$ and $C_{+} S^{0}$ are southern and northern hemi-circles. The clutching function $m: S^{0} \times F \rightarrow F$ is defined by

$$
g_{+} f_{-}(\{\varepsilon\}, x)=(\{\varepsilon\}, m(\{\varepsilon\}, x)), \quad \varepsilon=0,1 .
$$

Then $m \mid\{0\} \times F$ is homotopic to the map $(\{0\}, x) \rightarrow x$.

Now Spanier has shown that the top row is exact in the following diagram

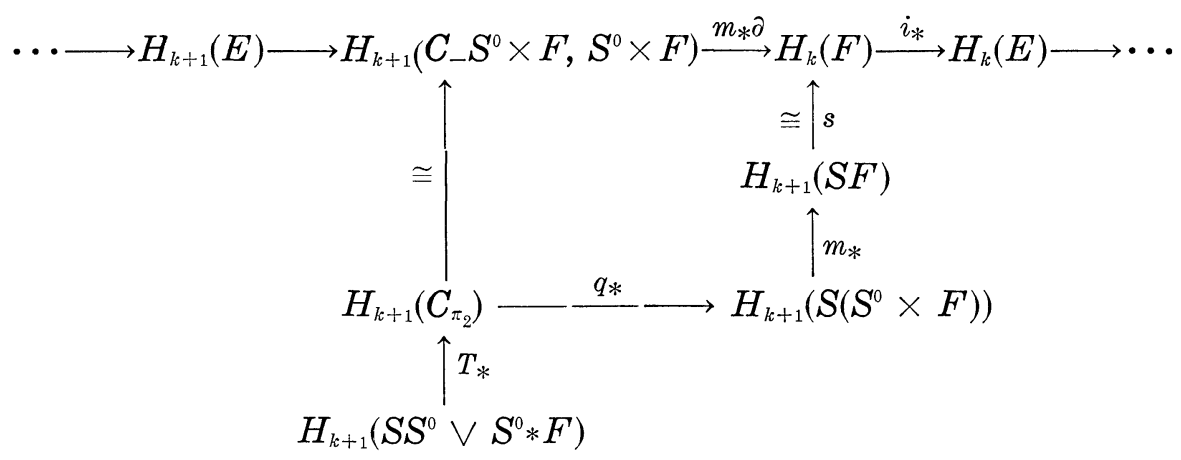

which is commutative up to sign, where $s$ is the suspension isomorphism, $\pi_{2}: S^{0} \times F \rightarrow F$ the projection, $q$ the map pinching $F$ to a point and $T: S S^{0} \vee S^{0} * F \rightarrow C_{\pi_{2}}$ denotes the homotopy equivalence defined in [7; 2.2]; thus, $m q T \mid S S^{0}$ is homotopic to the map $(\varepsilon, t) \rightarrow(m(\varepsilon, *), t)$ and $m q T \mid S^{0} * F$ is homotopic to the map $(1-t) \in \bigoplus t x \rightarrow(m(\varepsilon, x), t)$. Hence, using the homeomorphism $h: S F \rightarrow S^{0} * F$ given by

$$
h(x, s)= \begin{cases}(1-2 s)\{0\} \oplus 2 s x & 0 \leqq 2 s \leqq 1 \\ (2 s-1)\{1\} \oplus(2-2 s) x & 1 \leqq 2 s \leqq 2\end{cases}
$$

we see that $m q T h$ induces the homomorphism

$$
H_{k+1}(S F) \stackrel{(1-S \bar{m})_{*}}{\longrightarrow} H_{k+1}(S F),
$$

where $\bar{m}: F \rightarrow F$ denotes the map given by $\bar{m}(x)=m(\{1\}, x)$.

Consequently, the proof of Theorems 3 will be completed if the following assertion is proved:

$$
\bar{m}_{*}=\theta(e)_{*}
$$

Proof of (5). Observe that $+1 \in Z$ is represented by a loop $\omega$ in $S S^{0}=C_{+} S^{0} \cup C_{-} S^{0}$ which emanates at $\{0\}$. By considering $g_{+} f_{-}$ 
followed by a fiber homotopy inverse $f_{+}$of $g_{+}$, we infer easily that $\omega$ is lifted to a path $\tilde{\omega}_{x}$, depending continuously on $x \in F$, with $\tilde{\omega}_{x}(1)=$ $x$ and such that the map $x \rightarrow \tilde{\omega}_{x}(0)$ is homotopic to the map $x \rightarrow \bar{m}(x)$. Hence the definition of the action of the fibration and Lemma 1.3 imply the assertion (5).

\section{REFERENCES}

1. H. Bass, $K_{2}$ and symbols, Lecture Notes in Math. 108, Springer-Verlag, BerlinHeidelberg-New York, 1969.

2. B. Eckman and P. J. Hilton, Operators and cooperators in homotopy theory, Math. Ann., 141 (1960), 1-21.

3. T. Ganea, A generalization of the homology and homotopy suspension, Comment. Math. Helv., 39 (1965), 295-322.

4. T. Ganea, Homologie et extensions centrales de groupes, C. R. Acad. Sci. Paris, 266 (1968), 556-558.

5. I. M. Hall, The generalized Whitney sums, Quart. J. Math. Oxford Ser. (2), 16 (1965), 360-384.

6. M. A. Kervaire, Mulitiplicateurs de Schur et K-theorie, Essays on topology and related topics, Springer-Verlag. Berlin-Heidelberg-New York, 1970.

7. Y. Nomura, The Whitney join and its dual, Osaka J. Math., 7 (1970), 353-373.

8. E. H. Spanier, Algebraic topology, McGraw-Hill, New York, 1966.

Received March 10, 1971.

College of General Education, Osaka University 


\section{PACIFIC JOURNAL OF MATHEMATICS}

\section{EDITORS}

H. SAMELSON

Stanford University

Stanford, California 94305

C. R. Hовву

University of Washington

Seattle, Washington 98105
J. DugundJI

Department of Mathematics

University of Southern California

Los Angeles, California 90007

RICHARD ARENS

University of California

Los Angeles, California 90024

\section{ASSOCIATE EDITORS}
E. F. BECKENBACH
B. H. NeumanN
F. WOLF
K. YOSHIDA

\section{SUPPORTING INSTITUTIONS}

UNIVERSITY OF BRITISH COLUMBIA

CALIFORNIA INSTITUTE OF TECHNOLOGY

UNIVERSITY OF CALIFORNIA

MONTANA STATE UNIVERSITY

UNIVERSITY OF NEVADA

NEW MEXICO STATE UNIVERSITY

OREGON STATE UNIVERSITY

UNIVERSITY OF OREGON

OSAKA UNIVERSITY
UNIVERSITY OF SOUTHERN CALIFORNIA

STANFORD UNIVERSITY

UNIVERSITY OF TOKYO

UNIVERSITY OF UTAH

WASHINGTON STATE UNIVERSITY

UNIVERSITY OF WASHINGTON

$\stackrel{*}{*} \stackrel{*}{*} \stackrel{*}{*}{ }^{*}$ AMERICAN MATHEMATICAL SOCIETY

NAVAL WEAPONS CENTER 


\section{Pacific Journal of Mathematics}

\section{Vol. 41, No. $1 \quad$ November, 1972}

Anatole Beck and Peter Warren, Weak orthogonality.................

Jonnie Bee Bednar and Howard E. Lacey, Concerning Banach spaces whose duals are abstract $L$-spaces.............................

Louis Harvey Blake, Canonical extensions of measures and the extension of regularity of conditional probabilities..........................

R. A. Brooks, Conditional expectations associated with stochastic processes ..........................................

Theodore Allen Burton and Ronald Calvin Grimmer, On the asymptotic behavior of solutions of $x^{\prime \prime}+a(t) f(x)=e(t) \ldots \ldots \ldots \ldots \ldots \ldots$

Stephen LaVern Campbell, Operator-valued inner functions analytic on the closed disc ............................................

Yuen-Kwok Chan, A constructive study of measure theory...

Alexander Munro Davie and Bernt Karsten Oksendal, Peak interpolation sets for some algebras of analytic functions ...................

H. P. Dikshit, Absolute total-effective $\left(N, p_{n}\right)(c, 1)$ method ...............

Robert E. Edwards, Edwin Hewitt and Kenneth Allen Ross, Lacunarity for

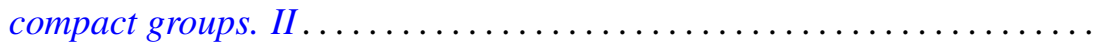

James Daniel Halpern, On a question of Tarski and a maximal theorem of Kurepa

Gerald L. Itzkowitz, A characterization of a class of uniform spaces that admit an invariant integral

Mo Tak Kiang, Semigroups with diminishing orbital diameters ..

Glenn Richard Luecke, A class of operators on Hilbert space ...

R. James Milgram, Group representations and the Adams spectral sequence. . .

G. S. Monk, On the endomorphism ring of an abelian p-group, and of a large subgroup...

Yasutoshi Nomura, Homology of a group extension ...

R. Michael Range, Approximation to bounded holomorphic functions on strictly pseudoconvex domains...

Norman R. Reilly, Inverse semigroups of partial transformations and $\theta$-classes.

Chris Rorres, Strong concentration of the spectra of self-adjoint operators

Saharon Shelah, A combinatorial problem; stability and order for models and theories in infinitary languages.

George Gustave Weill, Vector space decompositions and the abstract

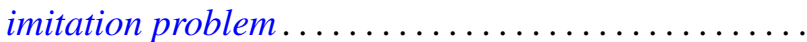

